\title{
BALLOON OBSERVATIONS OF GALACTIC COSMIC RAY HELIUM BEFORE AND DURING A FORBUSH DECREASE
}

\author{
J.M. Clem, T.G. Guzik, M. Lijowski, J.P. Wefel \\ Department of Physics and Astronomy, Louisiana State University \\ J.J. Beatty, D.J. Ficenec, S. Tobias \\ Physics Department, Washington University \\ J.W. Mitchell \\ NASA/Goddard Space Flight Center \\ S. McKee, S. Nutter, G. Tarle, A. Tomasch \\ Physics Department, University of Michigan \\ C.R. Bower, R.M. Heinz, S.L. Mufson, J. Musser, J. Pitts, G.M. Spiczak \\ Physics Department, Indiana University \\ S.P. Ahlen, B. Zhou \\ Physics Department, Boston University
}

\begin{abstract}
The energy spectrum of galactic cosmic ray Helium was measured in two different balloon experiments launched four days apart from Canada: SMILI-I on 1-Sept-1989 and MASS on 5-Sept-1989. A slow Forbush decrease began on 4-Sept-1989 and had not reached its maximum at the time of the MASS flight. Comparison of the balloon measurements shows a fractional decrease of 0.37 to 0.15 in the Helium flux between 200 and $450 \mathrm{MeV} /$ nucleon $(1.2-2.0 \mathrm{GV})$. The rigidity dependence is analyzed in two models and found to be steeper than previous observations. Interplanetary particle data and ground-based Neutron Monitor results are consistent with the balloon observations. Probable sources for this Forbush decrease are discussed.
\end{abstract}

\section{Introduction}

A Forbush decrease is characterized as a world-wide, abrupt decrease in the Neutron Monitor (NM) counting rate, followed by a slow recovery lasting a few days to several weeks (Lockwood, 1971). This effect is the result of a sudden change in the interplanetary medium near Earth, caused by shock wave turbulence, or moving magnetic clouds, which are interplanetary manifestations of a solar mass ejection or a high-velocity solar wind stream from a coronal hole. These interplanetary phenomena produce perturbations in the radially dependent diffusion coefficient which, in effect, sweeps back the Earthward-bound cosmic rays. Forbush decreases have been investigated for over three decades, but, due to the small geometry factor in most satellite detectors and to the high energy thresholds of ground-based neutron monitors, there is little information on the rigidity dependence of the effect, particularly below $2 \mathrm{GV}$.

A rare opportunity to observe the effects of a Forbush decrease on the Helium spectrum was provided when two balloon-borne cosmic ray detectors were launched from

Copyright 1993 by the American Geophysical Union.

Paper number 93GL01972

0094-8534/93/93GL-01972\$03.00
Prince Albert, Saskatchewan, Canada four days apart; the SMILI flight (Beatty et al., 1993) on 1-Sept-1989 and the MASS flight (Webber et al., 1991) on 5-Sept-1989. Instrumentation details can be found in Ahlen et al. (1991) and Webber et al. (1991). The common energy range measured was 200 to $2000 \mathrm{MeV} /$ nucleon (1.2-5.3 GV). On 4-Sept-1989, a slow Forbush decrease began, reaching a maximum on 7-Sept-1989 and not fully recovering before another Forbush decrease began on 18-Sept-1989. Employing the flux measured by the SMILI experiment as a reference, the measurement by MASS reflects the modulation during the Forbush decrease.

Over 30 years ago balloon measurements of the cosmic ray intensity were performed during Forbush decreases (McDonald et al, 1960; Meyer, 1960). Meyer (1960) determined the Helium counting rate in an integrating particle counter, with a top of the atmosphere threshold of $560 \mathrm{MeV} /$ nucleon, and showed that the ratio of the relative changes in the helium intensity to the relative changes in the Climax Neutron Monitor counting rate was constant.

McDonald et al. (1960) measured both proton and helium differential fluxes during and after a large Forbush decrease. Due to the low statistics and limited energy range in these observations, only a single proton interval in rigidity of $1.12-1.42 \mathrm{GV}$ did not have overlapping errors. These results were interpreted in terms of a modulation function $F(R) \propto R^{-\gamma}$ and were consistent with a relatively weak $(\gamma \sim 1)$ dependence. Note that this observation compared data during the recovery phase to postdecrease data, while the results presented here study the initial declining phase of the Forbush decrease.

\section{The Solar Terrestial Environment}

A type-II radio burst occurred on 2-Sept-1989 at 13:53 UT, as reported in the Solar-Geophysical Data reports (SGD). It was preceeded by four type-III radio bursts and two solar flares in $\mathrm{X}$-ray and $\mathrm{H} \alpha$ which occurred within a two hour period, as shown in Figure 1. The first $\mathrm{H} \alpha$ flare, located S17-18, E50-51, occurred 11:30-12:26 UT and was followed by a weaker second flare, located S20, 


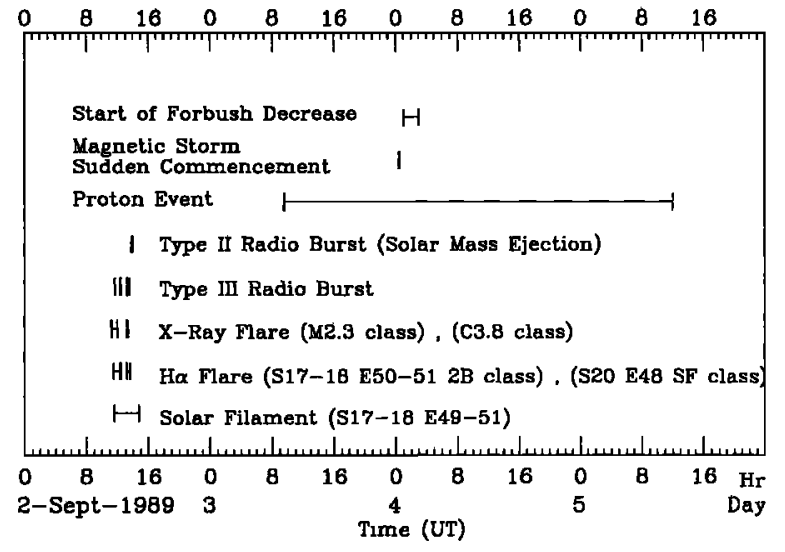

Fig. 1. Time sequence of solar-terrestrial events

E48, which occurred 13:15-13:36 UT. An active solar filament, located S17-18, E49-51, was reported at 11:3714:46 UT. The relative time and position correlations of the two $\mathrm{H} \alpha$ flares with an active filament strongly suggest association. It should be noted that all locations mentioned are within the same active region. The two $\mathrm{X}$-ray bursts along with the first and the fourth type-III radio bursts coincide with the two $\mathrm{H} \alpha$ flares. The type-II radio burst followed the latter event within 20 minutes, suggesting a Coronal Mass Ejection (CME).

The world-wide network of ground-base magnetometer measurements provides magnetic indices (e.g. Ap) which give the response of the magnetosphere to interplanetary magnetic field disturbances. An abrupt change in Ap usually indicates the occurrence of a magnetic storm sudden commencement (SC). The SC intensity indicates the strength of the disturbance, and the onset indicates the shock-front arrival time. The time difference between a type II solar radio burst and a SC represents the average shock transit time between leaving the solar corona and arriving at Earth. A type II solar radio burst is indicative of a shock wave moving upward through the solar corona usually preceeded by a type III solar radio burst and a solar flare in X-ray and $\mathrm{H} \alpha$ (Cane et al., 1984). A SC was observed at 00:27 UT on 4-Sept-1989 along with an abrupt change in the Ap index, suggesting the arrival of a shock front, which was then followed by the Forbush decrease. The time difference between the type II radio burst and the $\mathrm{SC}$ gives a transit time of 34.6 hours. Assuming the shock propagated radially, this implies an average velocity of $1200 \mathrm{~km} / \mathrm{sec}$.

A gradual solar proton event began at 09:30 UT on 3-Sept-1989 and reached a maximum at 04:30 UT on 4Sept-1989. The proton event, as observed in four channels at energies between 0.6 and $44 \mathrm{MeV}$ by the GOES-7 satellite, is shown at the top of Figure 2. This profile suggests that the particles were accelerated by a shock in the interplanetary medium. The location of the flares (Figure 1) on the East side of the solar disk is consistent with a gradual proton event (as contrasted with impulsive events observed most often from west side eruptions). The proton event is also consistent with a high speed solar wind stream, but there was no conclusive recurrence found 27 days prior to or following this event.

The significant feature of a shock wave is that it is an interplanetary region of high magnetic field which sets up a barrier to particle transmission, and one would expect a strong correlation between a shock-induced Forbush decrease and an Interplanetary Magnetic Field (IMF) dis- turbance and a solar wind speed enhancement (Lockwood et al., 1977). Unfortunately, IMF and solar wind measurements are unavailable for the 2-7 Sept. period.

The Forbush decrease is shown in Figure 2 as observed by the Climax and South Pole NM stations, which begin to show the decrease within the first few hours of 4-Sept-1989. The event can also be seen in the IMP8 high-energy RCK rate (protons $>105 \mathrm{MeV}$, courtesy University of Chicago). Note that IMP-8 was located anti-sunward $40 \mathrm{R}_{E}$ from the Earth during the decrease. The RCK and Neutron Monitor signals have been normalized to the mean observed during 1-Sept-1989.

In general, shock-waves are inclined with respect to the ecliptic plane resulting in a north-south anisotropy of the fractional intensity decrease measured by the NM stations. The directionality of the north-south anisotropy usually remains unchanged throughout recovery, assuming no further disturbances occur. The asymmetry during this Forbush decrease is indicated by the ratio of the Thule and McMurdo NM fractional changes as shown at the bottom of Figure 2. During 5 -Sept the Thule station observed a larger decrease than McMurdo suggesting that the shock arrived from north of the ecliptic. A northsouth phase inversion occurred on 7-Sept-1989, with an associated SC, a possible signature of a second shock arrival (Duggal et al, 1975).

\section{Observations and Interpretation}

The Helium Spectra observed in the SMILI and MASS experiments are shown in Figure 3. During 1989, solar activity was high and heliospheric modulation was strong as indicated by the solid curve which shows the helium spectrum modulated to $1200 \mathrm{MV}$ (diffusion coefficient = $\left.39.3 \times 10^{20}\left(\mathrm{~cm}^{2} / \mathrm{sec} \mathrm{GV}\right) \exp ((\mathrm{r}-1) / 29) \beta \mathrm{R}\right)$ using the full solution to the Fokker-Planck equation and a local interstellar spectrum in the form of $\mathrm{dF} / \mathrm{dE} \sim \mathrm{R}^{-2.68}$ (Fisk, 1971; Beatty, et al., 1993; Seo, et al., 1991). The modulated spectrum agrees well with the SMILI measurement and with the high energy MASS data, but the pronounced difference below $\sim 600 \mathrm{MeV} /$ nucleon shows

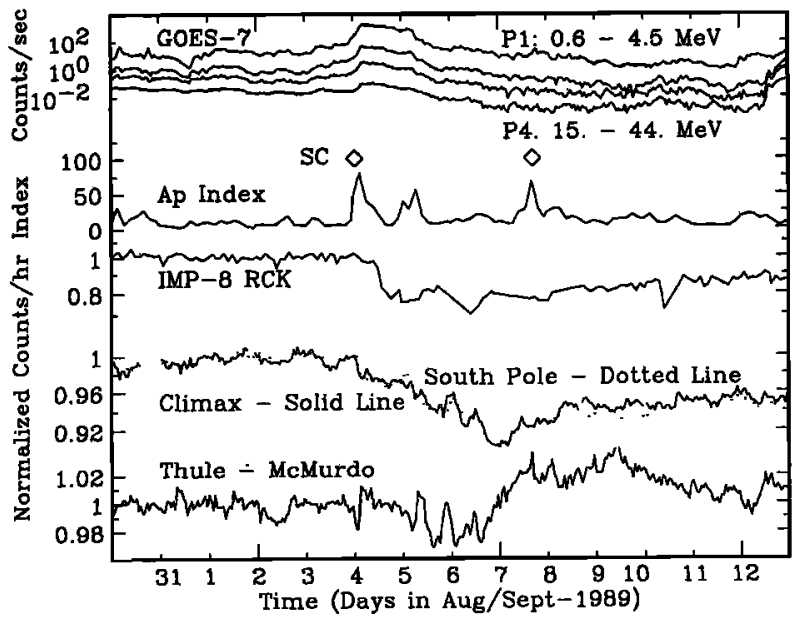

Fig. 2. GOES-7 Solar particle counts, Magnetic Storm Sudden Commencement, Ap index, IMP-8 RCK rate, Neutron Monitor rates and North-South Neutron Monitor ratio for 30-Aug-1989 through 12-Sept-1989. The tick marks represent 00:00 UT. 
the effect of the Forbush decrease. There is no heliospheric modulation level which can reproduce the MASS data over the full energy range assuming a linear betarigidity dependence in the diffusion coefficient. For the MASS flight, approximately one day after the Forbush decrease began, the Climax Neutron monitor had decreased to 0.95 of its value on 1-Sept. The MASS flux at $200 \mathrm{MeV} /$ nucleon shows a fractional decrease of $37 \%$ while at $450 \mathrm{MeV} /$ nucleon the fractional decrease is $15 \%$, a change of over a factor of two in an interval of rigidity of about $800 \mathrm{MV}$.

At high energy, the SMILI flux integrated over 1.2-2 $\mathrm{GeV} /$ nucleon is $20.1 \pm 1.4\left(\mathrm{~m}^{2} \mathrm{sr} \mathrm{s}\right)^{-1}$ while the MASS value is $23.0 \pm 1.8\left(\mathrm{~m}^{2} \mathrm{sr} \mathrm{s}\right)^{-1}$ giving a fractional difference of $14 \pm 12 \%$. Meyer (1960) found that the fractional change in the $>560 \mathrm{MeV} /$ nucleon Helium intensity was 1.3 times as large as the Climax NM fractional change during his Forbush decrease. This implies that we would expect a decrease of $6.5 \%$, which is not observed.

The difference in the integrated flux at high energy might represent a normalization shift between the two experiments, but this is difficult to confirm within the uncertainties (Note the large deviation of the MASS point at $\sim 1.2 \mathrm{GeV} /$ nucleon.) We will consider this normalization question below. Of more concern is the possibility of an energy dependent efficiency and/or an energy assignment uncertainty. The MASS flight results and those from LEAP, a previous flight of essentially the same instrument, agree at $10 \mathrm{GeV} /$ nucleon where modulation should be negligible. The techniques used to extract the spectra from MASS and LEAP (Seo, et al., 1991) were identical, and the LEAP results gave a helium spectrum consistent with other solar minimum measurements. In fact, it is the LEAP analysis that provided the local interstellar spectrum used to obtain the modulated curve in Figure 3. Thus, there is no reason to suspect any energy dependent normalization effects in the datasets.

In analyzing the rigidity dependence of the decrease, an often used form is a power law, $R^{-\gamma}$ (Flückinger 1985). The fractional decrease was determined using a linear interpolation of the measured Helium rigidity spectra to acquire common rigidity bins and is shown in Figure 4. In the 1.2-2.0 GV range the dependence (solid line) has

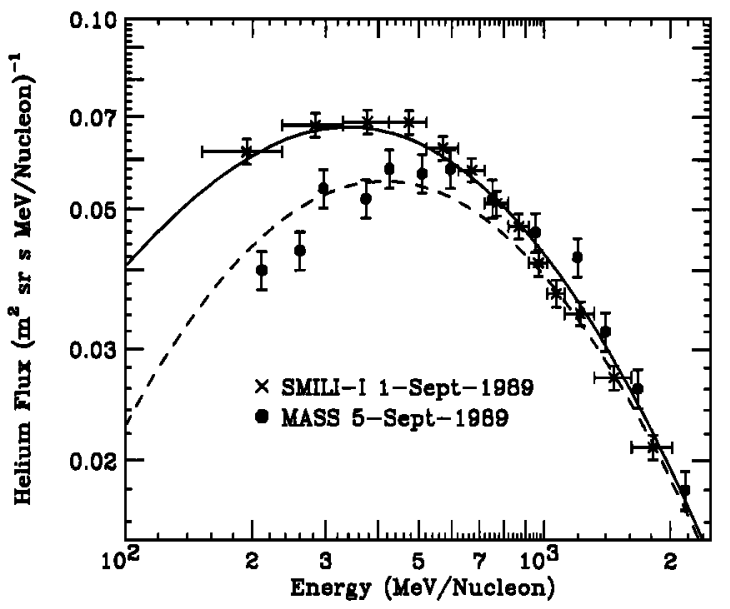

Fig. 3. Helium Energy Spectra measured by SMILI and MASS compared to calculated curves (see text for details).

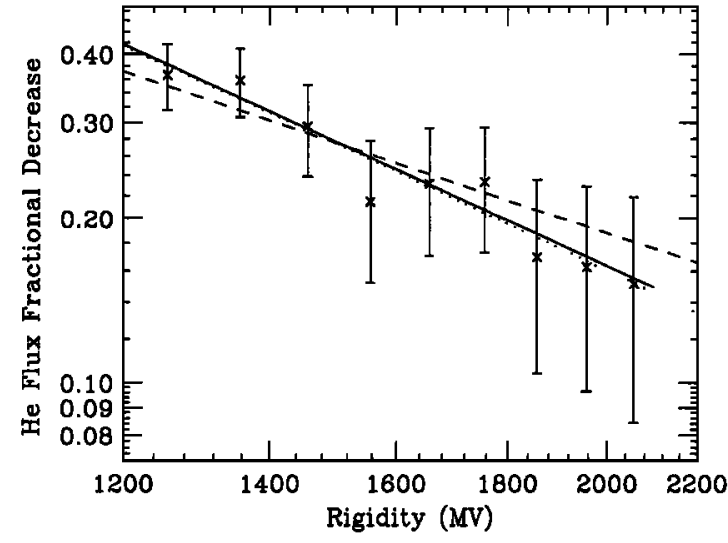

Fig. 4 The fractional decrease as a function of rigidity determined from the Helium rigidity spectra measured by SMILI and MASS. The curves are described in the text.

an exponent of $1.8 \pm 0.5(-0.2)$; steeper than the $R^{-1}$ form reported previously. The value in parenthesis is the additional uncertainty that results from allowing a $14 \%$ shift in the overall normalization between the two experiments.

McDonald et al. (1960) reported a fractional decrease in the proton flux of $65 \%$ within the interval 1.12-1.42 GV during a large Forbush decrease which produced a fractional decrease of $11 \%$ at Climax. Scaling to our Climax decrease of $5 \%$, predicts a decrease of $30 \%$. For SMILIMASS in the same rigidity interval, the observation is $37 \%$, consistent with the 1960 observations. (If the $14 \%$ normalization shift is included, the present observation is 46\%.) Further, the errors on the measured Helium flux reported by McDonald et al. (1960), suggest an upper-limit for the fractional decrease of 0.75 , at the $95 \%$ confidence level, within the range of 1.08-1.88 GV, again in agreement when scaled to the SMILI-MASS observations.

The IMP-8 RCK detector observed a fractional decrease of 0.22 . Assuming a typical solar maximum proton spectrum, the average energy of the particles detected by RCK is about $4.5 \mathrm{GeV}(5.4 \mathrm{GV})$, approximately the effective primary threshold of the Climax NM, and about $70 \%$ of the detected particles have a lower energy than the average. We estimate a fractional decrease of about 0.3 for protons between $105 \mathrm{MeV}$ and $4.5 \mathrm{GeV}$, equivalent, in rigidity, to Helium from $30-2000 \mathrm{MeV} /$ nucleon. Extrapolating the rigidity dependence measured for the SMILI-MASS data down to $30 \mathrm{MeV} /$ nucleon gives a predicted fractional decrease comparable to that observed in the RCK rate.

The present results can also be analyzed in a FokkerPlanck formalism (force-field approximation). Neglecting latitude-dependent effects and adiabatic deceleration and assuming a diffusion coefficient $=C \beta R^{\gamma}$, where $C$ is a constant, the solution can be written as (Fisk, 1971)

$$
\mathrm{J}_{1}=\mathrm{J}_{0} \exp \left(-\frac{3 \psi}{\beta \mathrm{R}^{\gamma}}\right)
$$

with a "local modulation parameter", $\psi$. The fit of this equation is shown as the the dotted line in Figure 4 and implies a value of $\gamma=1.6 \pm 0.5$, in agreement with the solid curve. For comparison, the fit with $\gamma=1$ is shown as the dashed line, which does not reproduce the measurements as well. 
To analyze the modulating effect of this Forbush decrease, we employ a solution to the full Fokker-Planck equation and assume that (a) the modulation takes place between 1.25 and 1.0 A.U., (b) the input helium spectrum is given by the solid curve in Figure 3 and (c) the diffusion coefficient is $C \beta R^{\gamma}$ with $\gamma=1.8$ and $C=2.2 \times 10^{20}\left(\mathrm{~cm}^{2} / \mathrm{sec}\right.$ $\left.\mathrm{GV}^{1.8}\right)$. The result is plotted as the dashed curve in Figure 3 and reproduces qualitatively the shape of the MASS data. (A similar result is obtained for $\gamma=1.6$.)

\section{Conclusion}

The SMILI and MASS flights provided a new measurement of the rigidity dependence of the decrease in the galactic cosmic ray Helium intensity, within an energy regime previously unexplored in detail. The observed intensity decrease is consistent with other measurements made during this time, and suggests, in the $1.2-2.0 \mathrm{GV}$ range, a power law index $\gamma \sim 1.8$ in the simplest modulation function $F(R) \propto R^{-\gamma}$, notably larger than reported previously (Flückinger, 1985). In the force-field FokkerPlanck analysis, $\gamma \sim 1.6$ is indicated. The present observations apply to the declining phase of the Forbush decrease and indicate stronger modulation for low rigidity particles compared to the recovery phase studied previously.

Acknowledgements. This work was supported by NASA grants NAGW-2138 (BU), 1032 (BU), 1035 (IU), 1027 (LSU), 1642 (UM), 2990 (WU) and the NASA Graduate Student Researchers Program (IU). Data used in this study were provided by the Solar-Terrestrial Physics division of NOAA, the University of Chicago, the National Space Science Data Center and the Bartol Research Institute. We would like to personally thank Jiasheng Chen, John Cooper, Paul Evenson, Roger Pyle, Yeming Sang and Herb Sauer for valuable discussions.

\section{References}

Ahlen, S., et al., SMILI (Superconducting Magnet Instrument for Light Isotopes) A Balloon Borne Magnet Spectrometer, 22nd ICR Conference Papers, 2, 563, 1991

Beatty, J.J., et al., The Cosmic Ray ${ }^{3} \mathrm{He} /{ }^{4} \mathrm{He}$ Ratio from $100 \mathrm{MeV} / \mathrm{amu}$ to $1600 \mathrm{MeV} / \mathrm{amu}$, Astrophysical Journal, in press, (August 10 issue) 1993
Cane, H.V., et al., Type-II Solar Radio Bursts, Interplanetary Shocks, and Energetic Particle Bursts, Astrophysical Journal, 282, 339, 1984

Duggal, S.P., et al., Interplanetary Shock Waves and the North-South Anisotropy in Cosmic Rays, 14th ICR Conference Papers, 4, 1176, 1975

Fisk, L.A., Solar Modulation of Galactic Cosmic Rays,2 Journal of Geophysical Research, 76, 221, 1971

Flückinger, E.O., Forbush Decreases, Geomagnetic and Atmospheric Effects, Cosmogenic Nuclides 19th ICR Conference Papers, 9, 301, 1985

Lockwood, J.A., Forbush decreases in Cosmic Radiation, Space Science Reviews, 12, 658, 1971

Lockwood, J.A., et al., Forbush Decreases and Interplanetary Magnetic Field Disturbances, Journal of Geophysical Research, 82, 1906, 1977

Meyer, P., et al., The Cosmic Ray Alpha-Particle Flux during Sharp Forbush Intensity Decreases, Journal of Geophysical Research, 65, 3881, 1960

McDonald, F.B., et al., Changes in the Low-Rigidity Primary Cosmic Radiation During the Large Forbush decrease of May 12, 1959 Journal of Geophysical Research, 65, 767, 1960

Seo, Eun-Suk, et al., Measurement of Galactic Cosmic Ray Proton and Helium Spectra During the 1987 Solar Minimum, Astrophysical Journal, 378 ,763, 1991

Webber,W.R. et al, A Measurement of the Cosmic-Ray ${ }^{2} \mathrm{H}$ and ${ }^{3} \mathrm{He}$ Spectra and ${ }^{2} \mathrm{H} /{ }^{4} \mathrm{He}$ and ${ }^{3} \mathrm{He} /{ }^{4} \mathrm{He}$ Ratios in 1989, Astrophysical Journal, 380, 230, 1991

J.M. Clem, T.G. Guzik, M. Lijowski and J.P. Wefel, Department of Physics and Astronomy, Louisiana State University, Baton Rouge, LA, 70803-4001

J.J. Beatty, D.J. Ficenec and S. Tobias Department of

Physics, Washington University, St. Louis, MO, 63130

S. McKee, S. Nutter, G. Tarle and A. Tomasch, Department of Physics, University of Michigan, Ann Arbor, MI, 48109-1120

C.R. Bower, R.M. Heinz, S.L. Mufson, J. Musser, J. Pitts and G.M. Spiczak, Department of Physics, Indiana University, Bloomington, IN, 47405

S.P. Ahlen and B. Zhou, Physics Department, Boston University, Boston, MA, 02215

J.W. Mitchell, Code 661, Goddard Space Flight Center, Greenbelt, MD, 20771

Received: March 11, 1993

Revised: June 4, 1993

Accepted: July 1, 1993 